\title{
SMOOTH, VERY SMOOTH AND STRONGLY SMOOTH POINTS IN MUSIELAK-ORLICZ SEQUENCE SPACES
}

\author{
Shurong Bian, Henryk Hudzik and Tingfu Wang
}

Criteria for smooth points, very smooth points and strongly smooth points in Musielak-Orlicz sequence spaces equipped with the Luxemburg norm are given.

\section{INTRODUCTION}

Let us denote by $X$ a real Banach space and by $S(X)$ the unit sphere of $X$. For any $x \in S(X)$ we denote by $\operatorname{Grad}(x)$ the set of all support functionals at $x$, that is, $\operatorname{Grad}(x)=\left\{f \in S\left(X^{*}\right): f(x)=\|x\|\right\}$, where $X^{*}$ denotes the dual space of $X$. A point $x \in S(X)$ is said to be a smooth point if $\operatorname{Grad}(x)$ is a singleton. A point $x \in S(X)$ is said to be a very smooth (strongly smooth or equivalently Frechet differentiable) point if it is a smooth point and for any sequence $\left(f_{n}\right)$ in $S\left(X^{*}\right)$ such that $f_{n}(x) \rightarrow 1$ we have $f_{n}-f \rightarrow 0$ weakly (respectively $\left\|f_{n}-f\right\| \rightarrow 0$ ), where $\{f\}=\operatorname{Grad}(x)$.

It is obvious that strong smoothness implies very smoothness and this implies smoothness. For these definitions and their applications we refer to [5].

A mapping $\Phi: \mathbb{R} \rightarrow[0, \infty]$ is said to be an Orlicz function if it is even, convex left-continuous on $[0, \infty), \Phi(0)=0$ and $\Phi(u)<\infty$ for some $u>0$ (see $[1,10,11,12$, $13,15])$. A sequence $M=\left(M_{i}\right)$ of Orlicz functions is called a Musielak-Orlicz function (see [14]). We associate with this function two sequences $\left(e_{i}\right)$ and $\left(b_{i}\right)$, where

$$
e_{i}=\sup \left\{u \geqslant 0: M_{i}(u)=0\right\}, \quad b_{i}=\sup \left\{u \geqslant 0: M_{i}(u)<\infty\right\}
$$

for each $i \in \mathbb{N}$. Moreover, $p_{i}^{-}(u)$ and $p_{i}(u)$ denote the left and the right derivative of $M_{2}$ at $u \in \mathbb{R}$ with $|u| \leqslant b_{i}$. Of course we assume $p_{i}\left(b_{i}\right)=\infty$ and $p_{i}^{-}(u)=p(u)=\infty$ for $u>b_{i}$.

If $N=\left(N_{i}\right)$ is the Musielak-Orlicz function complementary to $M=\left(M_{i}\right)$ in the sense of Young, that is, $N_{i}(v)=\sup _{u \geqslant 0}\left\{u|v|-M_{i}(u)\right\}$ for each $i \in \mathbb{N}$ and $v \in \mathbb{R}$, then we have Young's inequality

$$
|u v| \leqslant M_{i}(u)+N_{i}(v)
$$

Received 25th November, 1999

This work was supported by NSFC (19871020).

Copyright Clearance Centre, Inc. Serial-fee code: 0004-9727/01 \$A2.00+0.00. 
for every $u, v \in \mathbb{R}$, and for any $u \in \mathbb{R}$ the equality

$$
|u v|=M_{i}(u)+N_{i}(v)
$$

holds if and only if $p_{i}^{-}(u) \leqslant v \leqslant p_{i}(u)$.

We say a Musielak-Orlicz function $M=\left(M_{i}\right)$ satisfies the $\delta_{2}^{0}$-condition $\left(M \in \delta_{2}^{0}\right.$ for short) if there are positive constants $a$ and $k$, a natural number $i_{0}$ and a sequence $\left(c_{i}\right)_{i=i_{0}}^{\infty}$ with $c_{i} \geqslant 0$ such that $\sum_{i=i_{0}}^{\infty} c_{i}<\infty$ and the inequality

$$
M_{i}(2 u) \leqslant k M_{i}(u)+c_{i}
$$

holds for all $i \geqslant i_{0}$ and $u$ satisfying $M_{i}(u) \leqslant a$ (see [14]).

Let $l^{0}$ denote the space of all real sequences $x=(x(i))$. As usual, for $x \in l^{0}$, we denote supp $x=\{i \in \mathbb{N}: x(i) \neq 0\}$. With any Musielak-Orlicz function $M=\left(M_{i}\right)$ we associate the convex modular function $\rho_{M}: l^{0} \rightarrow[0, \infty]$ defined by

$$
\rho_{M}(x)=\sum_{i=1}^{\infty} M_{i}(x(i)) \quad\left(\forall x \in l^{0}\right)
$$

and the Musielak-Orlicz sequence space

$$
l_{M}=\left\{x \in l^{0}: \rho(x / \lambda)<\infty \text { for some } \lambda>0\right\} \text {. }
$$

In the space $l_{M}$ we define two norms; the Luxemburg norm

$$
\|x\|_{M}=\inf \left\{\lambda>0: \rho_{M}(x / \lambda) \leqslant 1\right\}
$$

and the Orlicz norm

$$
\|x\|_{M}^{0}=\sup \left\{\sum_{i=1}^{\infty} x(i) y(i): \rho_{N}(y) \leqslant 1\right\} .
$$

By $h_{M}$ we denote the subspace of $l_{M}$ which is defined to be the closure in $l_{M}$ of the space of all sequences in $l^{0}$ with finite number of coordinates different from 0 (the closure is taken in the norm topology). It is easy to see that

$$
h_{M}=\left\{x \in l^{0}: \text { for any } \lambda>0 \text { there is } i_{\lambda} \in \mathbb{N} \text { such that } \sum_{i \geqslant i_{\lambda}} M_{i}(\lambda x(i))<\infty\right\} .
$$

The spaces $l_{M}$ and $h_{M}$ are Banach spaces under either of these two norms (see $[1,11,14]$ ). In [11] these spaces are called modular sequence spaces. The function $d_{M}: l_{M} \rightarrow[0, \infty)$ defined by

$$
d_{M}(x)=\inf \left\{\lambda>0: \sum_{i \geqslant i_{0}} M_{i}\left(\frac{x(i)}{\lambda}\right)<\infty \text { for some } i_{0} \in \mathbb{N}\right\}
$$


is nothing but the distance of $x \in l_{M}$ from the subspace $h_{M}$ (see [1]).

Every $f \in \operatorname{Grad}(x)$ for $x \in l_{M} \backslash\{0\}$ is of the from $f=y+\varphi$, where $y \in l_{N}^{0}$ and $\varphi$ is a singular functional, that is, $\varphi(z)=0$ for any $z \in h_{M}$, and $y$ is identified with the functional

$$
\langle w, y\rangle=\sum_{i=1}^{\infty} w(i) y(i) \quad\left(\forall w=(w(i)) \in l_{M}\right) .
$$

If $\varphi=0$, we say that $f$ is a regular support functional at $x$. The set of all regular support functionals at $x$ is denoted by $R \operatorname{Grad}(x)$. It is well known that (see $[1,15]$ )

$$
\|f\|=\|y\|_{N}^{0}+\|\varphi\| .
$$

Smooth points and smoothness in Orlicz spaces and Musielak-Orlicz spaces has been discussed for both the Luxemburg and the Orlicz norm as well as for a non-atomic measure and for the counting measure in the papers $[2,3,4,6,7,8,9,10,17]$.

In $[8]$ the following theorem was presented.

ThEOREM 0.1 . Let $x \in S\left(l_{M}\right)$.

I If $|x(i)|<b_{i}$ for $i=1,2, \ldots$, then $x$ is smooth if and only if

(a) $d_{M}(x)<1$,

(b) Card $\left\{i \in \mathbb{N}: p_{i}(|x(i)|) \neq 0\right\}=1$ or $p_{i}^{-}(|x(i)|)=p_{i}(|x(i)|)$ $(i=1,2, \ldots)$.

II If $\left|x\left(i_{0}\right)\right|=b_{i_{0}}$ for some $i_{0} \in \mathbb{N}, \rho_{M}(x)=\sup \left\{\rho_{M}(y):\|y\|_{M}=1\right.$, supp $y \subset$ $\operatorname{supp} x\}$, then $x$ is smooth if and only if

(a) $|x(i)|<b_{i}$ for any $i \neq i_{0}$,

(b) $p_{i_{0}}^{-}\left(b_{i_{0}}\right)=\infty$ or $p_{i}(|x(i)|)=0$ for $i \neq i_{0}$ or $v \notin l_{N}$, where $v=\{v(i)\}, v(i) \in$ $\left[p_{i}^{-}(|x(i)|), p_{i}(|x(i)|)\right]$ for $i=1,2, \ldots$

(c) $d_{M}(x)<1$.

III If $\left|x\left(i_{0}\right)\right|=b_{i_{0}}$ for some $i_{0} \in \mathbb{N}, \rho_{M}(x)<\sup \left\{\rho_{M}(y):\|y\|_{M}=1, \operatorname{supp} y \subset\right.$ $\operatorname{supp} x\}$, then $x$ is smooth if and only if

(a) $|x(i)|<b_{i}, i \neq i_{0}$,

(b) $d_{M}(x)<1$.

The formulation of this theorem is too complicated and, as we shall see below, its part II is not true (the assumptions are not necessary in general). Indeed, if $M=\left(M_{i}\right)$, where

$$
M_{i}(u)= \begin{cases}|u| & \text { if }|u| \leqslant \frac{1}{2} \\ \infty & \text { if }|u|>\frac{1}{2}\end{cases}
$$

for each $i \in \mathbb{N}$, define $x=(1 / 2,0,0, \ldots)$. Obviously $\|x\|_{M}=1$. Since $x(1)=1 / 2=$ $b_{1}: \rho_{M}(x)=1 / 2=\sup \left\{\rho_{M}(y):\|y\|_{M}=1, \operatorname{supp} y \subset \operatorname{supp} x=\{1\}\right\}, \quad x$ belongs 
to case II. Since $p_{1}^{-}\left(b_{1}\right)=p_{1}^{-}(1 / 2)=1<\infty, p_{2}(x(2))=p_{2}(0)=1 \neq 0$, we have $\sum_{i=1}^{\infty} N_{i}\left(p_{i}^{-}(x(i))\right)=\sum_{i=1}^{\infty} N_{i}(1)=\sum_{i=1}^{\infty} 0=0$, whence $\left(p_{i}^{-}(x(i))\right) \in l_{N}^{0}$. So, condition (b) of case II is not satisfied, whence it follows that if case II of Theorem 0.1 is true, $x$ should be not a smooth point. However, we shall prove that $x$ is smooth. Since $d_{M}(x)=0<1$, $\operatorname{Grad}(x)$ contains only regular (that is; order continuous) functionals (see [1]). We show that if $y \in S\left(l_{N}^{0}\right)$ belongs to $\operatorname{Grad}(x)$, then $y(i)=0$ for any $i \neq 1$. Indeed, if $y(2)>0$, then for $\bar{x}=(1 / 2,1 / 2,0,0, \ldots) \in S\left(l_{M}\right)$, we have

$$
1 \geqslant\langle\bar{x}, y\rangle=\langle x, y\rangle+\frac{1}{2} y(2)=1+\frac{y(2)}{2}
$$

which means that $y \notin \operatorname{Grad}(x)$, a contradiction. Therefore $y(i)=0$ for any $i \neq 1$ if $y \in \operatorname{Grad}(x)$, which means that $\operatorname{Grad}(x)$ is a singleton, that is, $x$ is a smooth point.

We shall establish a new criterion for smooth points in $S\left(l_{M}\right)$ and we shall also give criteria for very smooth points and strongly smooth points of $S\left(l_{M}\right)$.

Before proving new results let us recall some results concerning $l_{M}$ that will be used in this paper.

LEMMA 0.1. For each $x \in l_{M}, d\left(x, h_{M}\right)=d_{M}(x)$ (see [1, Theorem 1.4.3]).

LEMMA 0.2 . If $x \in l_{M}$ and $d_{M}(x)<1$, then $\operatorname{Grad}(x)=R \operatorname{Grad}(x)(\operatorname{see}[7$, Lemma 1.7]).

Lemma 0.3. If $x \in S\left(l_{M}\right)$ and $d_{M}(x)=1$, then there exist $y, z \in S\left(l_{M}\right)$ with $\operatorname{supp} x \cap \operatorname{supp} y=\emptyset$ and $y+z=x$ (see [8, Proposition 1]).

LEMma 0.4 . If $x \in S\left(l_{M}\right)$ and $|x(i)|=b_{i},|x(j)|=b_{j}$ for $i \neq j$, then there exist $y, z \in S\left(l_{M}\right)$ such that $\operatorname{supp} y \cap \operatorname{supp} z=\emptyset$ and $y+z=x$ (see [8, Proposition 2]).

Lemma 0.5. Assume that $y \in l_{N}^{0} \backslash\{0\}$. Then

(i) $\|y\|_{N}^{0}=\left(1+\rho_{N}(k y)\right) / k$ for some $k>0$ whenever $\sum_{i \in \text { supp } y} M_{i}\left(b_{i}\right)>1$,

(ii) $\|y\|_{N}^{0}=\sum_{i=1}^{\infty} b_{i}|y(i)|$ whenever $\sum_{i \in \text { supp } y} M_{i}\left(b_{i}\right) \leqslant 1$ (see [16]).

\section{RESULTS}

First we prove some auxiliary lemmas.

Lemma 1.1. If $M=\left(M_{i}\right)$ is a Musielak-Orlicz function such that $N \in \delta_{2}^{0}$ and $\left(y_{n}\right)$ is a sequence in $l_{N}^{0}$ such that $y_{n}(i) \rightarrow 0$ for each $i \in \mathbb{N}$ and

$$
\lim _{i_{0} \rightarrow \infty} \sup _{n} \sum_{i>i_{0}} N_{i}\left(y_{n}(i)\right)=0
$$

then $\left\|y_{n}\right\|_{N}^{0} \rightarrow 0$ 
Proof: Choose any $\varepsilon>0$ and take $h>0$ such that $4 / h<\varepsilon$. By $N \in \delta_{2}^{0}$, there exist $k>0, a>0, i_{0} \in \mathbb{N}$ and $c_{i} \geqslant 0\left(i>i_{0}\right)$ with $\sum_{i>i_{0}} c_{i}<\infty$ such that

$$
N_{i}(h v) \leqslant k N_{i}(v)+c_{i} \text { if } i>i_{0}, N_{i}(v) \leqslant a
$$

Without loss of generality we assume that $\sum_{i>i_{0}} c_{i} \leqslant 1$. By (1), there is $i_{0}^{\prime}>i_{0}$ such that

$$
\sup _{n} \sum_{i>i_{0}^{\prime}} N_{i}\left(y_{n}(i)\right) \leqslant \min \left\{a, \frac{1}{k}\right\} \text {. }
$$

By $y_{n}(i) \rightarrow 0$ for each $i \in \mathbb{N}$, we get $\sum_{i=1}^{i_{0}^{\prime}} N_{i}\left(h y_{n}(i)\right) \leqslant 1$ for $n$ large enough. Hence, we get

$$
\begin{aligned}
\left\|y_{n}\right\|_{N}^{0} & \leqslant \frac{1}{h}\left(1+\rho_{N}\left(h y_{n}\right)\right)=\frac{1}{h}\left(1+\sum_{i=1}^{i_{0}^{\prime}} N_{i}\left(h y_{n}(i)\right)+\sum_{i>i_{0}^{\prime}} N_{i}\left(h y_{n}(i)\right)\right) \\
& \leqslant \frac{1}{h}\left(2+\sum_{i>i_{0}^{\prime}}\left(k N_{i}\left(y_{n}(i)\right)+c_{i}\right)\right) \leqslant \frac{1}{h}\left(2+k \cdot \frac{1}{k}+1\right)<\varepsilon
\end{aligned}
$$

for $n$ large enough, which means that $\left\|y_{n}\right\|_{N}^{0} \rightarrow 0$ as $n \rightarrow \infty$.

LEMmA 1.2. If $\|x\|_{M}=1, d_{M}(x)<1-\theta<1, y_{n} \in S\left(l_{N}^{0}\right)$ for any $n \in \mathbb{N}$ and $\left\langle x, y_{n}\right\rangle \rightarrow 1$ as $n \rightarrow \infty$, then condition (1) from Lemma 1.1 holds.

Proof: If (1) is not true, by passing to a subsequence if necessary, we may assume that there are a sequence $\left(i_{n}\right) \subset \mathbb{N}$ with $i_{n} \nearrow \infty$ and $\varepsilon_{0}>0$ such that

$$
\sum_{i>i_{n}} N_{i}\left(y_{n}(i)\right) \geqslant \varepsilon_{0} \quad(n=1,2, \ldots) .
$$

We consider two cases.

I. $\left\|y_{n}\right\|_{N}^{0}=1 / k_{n}\left(1+\rho_{N}\left(k_{n} y_{n}\right)\right)$ for an infinite number of $n$. Noticing that $k_{n} \geqslant 1$, we get a contradiction:

$$
\begin{aligned}
1 \leftarrow & \left\langle x, y_{n}\right\rangle \\
& =\frac{1}{k_{n}}\left\langle x, k_{n} y_{n}\right\rangle \\
& =\frac{1}{k_{n}}\left(\sum_{i=1}^{i_{n}} k_{n} x(i) y_{n}(i)+(1-\theta) \sum_{i>i_{n}} \frac{x(i)}{1-\theta} y_{n}(i)\right) \\
& \leqslant \frac{1}{k_{n}}\left(\sum_{i=1}^{i_{n}}\left(M_{i}(x(i))\right)+N_{i}\left(k_{n} y_{n}(i)\right)+(1-\theta) \sum_{i>i_{n}}\left(M_{i}\left(\frac{x(i)}{1-\theta}\right)+N_{i}\left(k_{n} y_{n}(i)\right)\right)\right) \\
& \leqslant \frac{1}{k_{n}}\left(\rho_{M}(x)+\rho_{N}\left(k_{n} y_{n}\right)-\theta \sum_{i>i_{n}} N_{i}\left(k_{n} y_{n}(i)\right)+(1-\theta) \sum_{i>i_{n}} M_{i}\left(\frac{x(i)}{1-\theta}\right)\right)
\end{aligned}
$$




$$
\begin{aligned}
& \leqslant \frac{1}{k_{n}}\left(1+\rho_{N}\left(k_{n} y_{n}\right)-\theta \varepsilon_{0}+\sum_{i>i_{n}} M_{i}\left(\frac{x(i)}{1-\theta}\right)\right) \\
& \leqslant\left\|y_{n}\right\|_{N}^{0}-\theta \varepsilon_{0}+\sum_{i>i_{n}} M_{i}\left(\frac{x(i)}{1-\theta}\right) \rightarrow 1-\theta \varepsilon_{0} .
\end{aligned}
$$

II. $\left\|y_{n}\right\|_{N}^{0}=\sum_{i=1}^{\infty} b_{i}\left|y_{n}(i)\right|$ for an infinite number of $n$.

By $d_{M}(x)<1-\theta$, there is $i_{0} \in \mathbb{N}$ such that $\sum_{i>i_{0}} M_{i}(x(i) /(1-\theta))<\infty$. So, $|x(i)| /(1-\theta) \leqslant b_{i}$ for $i>i_{0}$. From

$$
N_{i}\left(y_{n}(i)\right)=\int_{0}^{\left|y_{n}(i)\right|} q_{i}(s) d s \leqslant \int_{0}^{\left|y_{n}(i)\right|} b_{i} d s=b_{i}\left|y_{n}(i)\right|
$$

where $q_{i}(s)=\sup \left\{t \geqslant 0: p_{i}(t) \leqslant s\right\}$, we have $\sum_{i>i_{n}} b_{i}\left|y_{n}(i)\right| \geqslant \varepsilon_{0}$. Hence for $i_{n}>i_{0}$, we get a contradiction:

$$
\begin{aligned}
1 \leftarrow\left\langle x, y_{n}\right\rangle & =\sum_{i=1}^{i_{n}} x(i) y_{n}(i)+(1-\theta) \sum_{i>i_{n}} \frac{x(i)}{1-\theta} y_{n}(i) \\
& \leqslant \sum_{i=1}^{i_{n}} b_{i}\left|y_{n}(i)\right|+(1-\theta) \sum_{i>i_{n}} b_{i}\left|y_{n}(i)\right|=\sum_{i=1}^{\infty} b_{i}\left|y_{n}(i)\right|-\theta \sum_{i>i_{n}} b_{i}\left|y_{n}(i)\right| \\
& \leqslant\left\|y_{n}\right\|_{N}^{0}-\theta \varepsilon_{0}=1-\theta \varepsilon_{0} .
\end{aligned}
$$

The following lemma is very important in the remaining considerations.

LEMMA 1.3. If $\|x\|_{M}=1,\left\|y_{n}\right\|_{N}^{0}=1$ for each $n \in \mathbb{N}$ and $\left\langle x, y_{n}\right\rangle \rightarrow 1$ as $n \rightarrow \infty$, then:

(i) $y_{n}(j) \rightarrow 0$ as $n \rightarrow \infty$ whenever $|x(j)|<e_{j}$,

(ii) $\liminf _{n \rightarrow \infty}\left(y_{n}(i) p_{j}(x(j))-y_{n}(j) p_{i}^{-}(x(i))\right) \geqslant 0$ whenever $|x(j)| \geqslant e_{j}$ and $|x(i)|>0$.

Proof: We may assume without loss of generality that $x(i) \geqslant 0$ for any $i \in \mathbb{N}$. If (i) is not true, we may assume that there is $j \in \mathbb{N}$ such that $x(j)<e_{j}$ and $y_{n}(j) \geqslant c>0$ for each $n \in \mathbb{N}$. Let us define $\bar{x}$ with $\bar{x}(j)=e_{j}$ and $\bar{x}(i)=x(i)$ for $i \neq j$. It is easy to see that $\rho_{M}(\bar{x})=\rho_{M}(x) \leqslant 1$, whence $\|\bar{x}\|_{M}=1$. Hence

$$
\begin{aligned}
1 & \geqslant\|\bar{x}\|_{M}\left\|y_{n}\right\|_{N}^{0} \geqslant\left\langle\bar{x}, y_{n}\right\rangle=\left\langle x, y_{n}\right\rangle+y_{n}(j)\left(e_{j}-x(j)\right) \\
& \geqslant\left\langle x, y_{n}\right\rangle+c\left(e_{j}-x(j)\right) \rightarrow 1+c\left(e_{j}-x(j)\right)
\end{aligned}
$$

which is a contradiction. So (i) is proved.

If (ii) is not true, there are $x(j) \geqslant e_{j}, x(i)>0$ and $a>0$ satisfying

$$
y_{n}(i) p_{j}(x(j))<y_{n}(j) p_{i}^{-}(x(i))-2 a \quad(n=1,2, \ldots)
$$


Since $\rho_{N}\left(y_{n}\right) \leqslant\left\|y_{n}\right\|_{N} \leqslant\left\|y_{n}\right\|_{N}^{0}=1$, we get $\left|y_{n}(j)\right| \leqslant A_{j}:=\sup \left\{v \geqslant 0: N_{j}(v) \leqslant 1\right\}$ for any $j \in \mathbb{N}$. (Note that $A_{j}=N_{j}^{-1}(1)$ if $N_{j}\left(A_{j}\right)=1$ and $A_{j}=b_{j}$ if $N_{j}\left(A_{j}\right)<1$.) Since $p_{i}^{-}$ is left continuous and $p_{i}$ is right continuous one can find a number $r>0$ such that

$$
p_{i}^{-}(x(i))-p_{i}^{-}(x(i)-r)<\frac{a}{2 A_{j}}, \quad p_{j}(x(j)+r)-p_{j}(x(j))<\frac{1}{2 A_{i}} .
$$

Then

$$
\left(p_{i}^{-}(x(i))-p_{i}^{-}(x(i)-r)\right) y_{n}(j)<\frac{a}{2}, \quad\left(p_{j}(x(j)+r)-p_{j}(x(j))\right) y_{n}(i)<\frac{a}{2} .
$$

Thus

$$
\begin{aligned}
y_{n}(i) p_{j}(x(j)+r) & <y_{n}(i) p_{j}(x(j))+\frac{a}{2}<y_{n}(j) p_{i}^{-}(x(i))-2 a+\frac{a}{2} \\
& <y_{n}(j) p_{i}^{-}(x(i)-r)+\frac{a}{2}-2 a+\frac{a}{2}=y_{n}(j) p_{i}^{-}(x(i)-r)-a
\end{aligned}
$$

for all $n \in \mathbb{N}$. We have

$$
\int_{x(j)}^{x(j)+\tau} p_{j}(s) d s>\int_{x(i)-r}^{x(i)} p_{i}^{-}(s) d s \text { or } \int_{x(j)}^{x(j)+r} p_{j}(s) d s \leqslant \int_{x(i)-r}^{x(i)} p_{i}^{-}(s) d s
$$

and we may assume that the second inequality holds. Denote $c_{j}=x(j)+r$. One can find a number $c_{i}, x(i)>c_{i} \geqslant x(i)-r$ such that

$$
\int_{x(j)}^{c_{j}} p_{j}(s) d s=\int_{c_{i}}^{x(i)} p_{i}^{-}(s) d s
$$

Of course

$$
y_{n}(i) p_{j}\left(c_{j}\right)<y_{n}(j) p_{i}^{-}\left(c_{i}\right)-a \quad(n=1,2, \ldots) .
$$

Since $0<p_{j}\left(c_{j}\right), p_{i}^{-}\left(c_{i}\right)<\infty$, there is $k>0$ such that

$$
\frac{y_{n}(i)}{p_{i}^{-}\left(c_{i}\right)}<k<\frac{y_{n}(j)}{p_{j}\left(c_{j}\right)}-\frac{a}{p_{i}^{-}\left(c_{i}\right) p_{j}\left(c_{j}\right)}(n=1,2, \ldots) .
$$

Define $\bar{x}$ with $\bar{x}(i)=c_{i}, \bar{x}(j)=c_{j}, \bar{x}(t)=x(t)$ for $t \neq i$ and $t \neq j$. Then

$$
\begin{aligned}
\rho_{M}(\bar{x}) & =\rho_{M}(x)+M_{j}\left(c_{j}\right)-M_{j}(x(j))+M_{i}\left(c_{i}\right)-M_{i}(x(i)) \\
& =\rho_{M}(x)+\int_{x(j)}^{c_{j}} p_{j}(s) d s-\int_{c_{i}}^{x(i)} p_{i}^{-}(s) d s=\rho_{M}(x) \leqslant 1,
\end{aligned}
$$

whence $\|\bar{x}\|_{M} \leqslant 1$. From (2) and (3), we get

$$
\begin{aligned}
1 & \geqslant\left\langle\bar{x}, y_{n}\right\rangle=\left\langle x, y_{n}\right\rangle+y_{n}(j)\left(c_{j}-x(j)\right)-y_{n}(i)\left(x(i)-c_{i}\right) \\
& =\left\langle x, y_{n}\right\rangle+\int_{x(j)}^{c_{j}}\left(y_{n}(j)-k p_{j}(s)\right) d s-\int_{c_{i}}^{x(i)}\left(y_{n}(i)-k p_{i}^{-}(s)\right) d s \\
& \geqslant\left\langle x, y_{n}\right\rangle+\int_{x(j)}^{c_{j}} \frac{a}{p_{i}^{-}\left(c_{i}\right)} d s=\left\langle x, y_{n}\right\rangle+\frac{a r}{p_{i}^{-}\left(c_{i}\right)} \rightarrow 1+\frac{a r}{p_{i}^{-}\left(c_{i}\right)}
\end{aligned}
$$


which is a contradiction finishing the proof.

As an immediate consequence of Lemma 1.3, we get

Lemma 1.4. Assume that $\|x\|_{M}=1,\|y\|_{N}^{0}=1$ and $\langle x, y\rangle=1$. Then:

(i) $y(j)=0$ whenever $|x(j)|<e_{j}$,

(ii) $y(i) p_{j}(x(j)) \geqslant y(j) p_{i}^{-}(x(i))$ whenver $|x(j)| \geqslant e_{j}$ and $|x(i)|>0$.

Now, we are ready to give criteria for smooth points of $S\left(l_{M}\right)$.

ThEOREM 1.1. If $x \in S\left(l_{M}\right)$, then $x$ is a smooth point if and only if:

(i) $d_{M}(x)<1$,

(ii) there is at most one index $i$ satisfying $|x(i)|=b_{i}$,

(iii-1) if $|x(i)|<b_{i}$ for all $i \in \mathbb{N}$, then $p_{i}^{-}(|x(i)|)=p_{i}(|x(i)|)$ whenever $|x(i)|<$ $M_{i}^{-1}(1)$

(iii-2) if $\left|x\left(i_{0}\right)\right|=b_{i_{0}}$ for some $i_{0} \in \mathbb{N}$ and $|x(i)|<b_{i}$ for $i \neq i_{0}$, then $\rho_{N}(x)<1$ or $p_{i_{0}}^{-}\left(b_{i_{0}}\right)=\infty$ or $p_{i}(|x(i)|)=0$ for $i \neq i_{0}$.

Proof: Assume without loss of generality that $x(i) \geqslant 0$ for all $i \in \mathbb{N}$.

NECESSITY. If (i) or (ii) is not true, then by Lemma 0.3 and Lemma 0.4 , there are $y, z \in S\left(l_{M}\right)$ such that supp $y \cap \operatorname{supp} z=\emptyset$ and $x=y+z$. Clearly, $y-z \in S\left(l_{M}\right)$ too. Take $y^{*} \in \operatorname{Grad}(y), z^{*} \in \operatorname{Grad}(z)$. Then

$$
1 \pm y^{*}(z)=y^{*}(y \pm z) \leqslant\left\|y^{*}\right\|\|y \pm z\|_{M}=\left\|y^{*}\right\|=1 .
$$

So $y^{*}(z)=0$. Similarly $z^{*}(y)=0$. Consequently $y^{*}(y)=z^{*}(z)=1$, whence $y^{*} \neq z^{*}$. Moreover

$$
y^{*}(x)=y^{*}(y+z)=y^{*}(y)=\|y\|_{M}=1 .
$$

So $y^{*} \in \operatorname{Grad}(x)$. Similarly, $z^{*} \in \operatorname{Grad}(x)$, which is a contradiction proving the necessity of (i) and (ii).

Now, we shall prove that $d_{M}(x)<1$ and $|x(i)|<b_{i}$ for all $i \in \mathbb{N}$ imply

$$
\rho_{M}(x)=1
$$

and

$$
\rho_{N}\left(p^{-}(x)\right) \leqslant \sum_{i=1}^{\infty}|x(i)| p_{i}^{-}(|x(i)|)<\infty .
$$

Indeed, by $d_{M}(x)<1$, there exists $\lambda>1$ and $i_{0} \in \mathbb{N}$ such that $\sum_{i>i_{0}} M_{i}(\lambda x(i))<\infty$. By $x(i)<b_{i}$, there exists $\lambda_{0}$ with $1<\lambda_{0} \leqslant \lambda$ such that $\sum_{i=1}^{i_{0}} M_{i}\left(\lambda_{0} x(i)\right)<\infty$. Hence $\rho_{M}\left(\lambda_{0} x\right)<\infty$. Since $\rho_{M}(t x)$ is a continuous function of $t$ on the interval $\left[0, \lambda_{0}\right]$, we obtain (4). From

$$
M_{i}\left(\lambda_{0} u\right) \geqslant \int_{u}^{\lambda_{0} u} p_{i}(s) d s \geqslant\left(\lambda_{0}-1\right) u p_{i}^{-}(u)
$$


and $\rho_{M}\left(\lambda_{0} x\right)<\infty$, we get (5).

If (iii-1) does not hold, we may assume that $p_{1}^{-}(x(1))<p_{1}(x(1))$ and $M_{1}(x(1))<1$. By (4), there is $i \in \mathbb{N}$ (we may assume that $i=2$ ) such that $M_{2}(x(2))>0$. Therefore $p_{2}^{-}(x(2))>0$ and, by (5),

$$
\sum_{i=1}^{\infty} x(i) p_{i}^{-}(x(i))<\infty \text { and } \sum_{i=2}^{\infty} x(i) p_{i}^{-}(x(i))+x(1) p_{1}(x(1))<\infty .
$$

Let $x^{*}, \bar{x}^{*}$ be defined as follows:

$$
\begin{aligned}
& x^{*}(i)=\frac{p_{i}^{-}(x(i))}{\sum_{i=1}^{\infty} x(i) p_{i}^{-}(x(i))} \quad(i=1,2, \ldots,), \\
& \bar{x}^{*}(i)= \begin{cases}\frac{p_{1}(x(1))}{\sum_{i \neq 1} x(i) p_{i}^{-}(x(i))+x(1) p_{1}(x(1))} & \text { if } i=1 \\
\frac{p_{i}^{-}(x(i))}{\sum_{i \neq 1} x(i) p_{i}^{-}(x(i))+x(1) p_{1}(x(1))} & \text { if } i \neq 1 .\end{cases}
\end{aligned}
$$

Since $\left\langle x, x^{*}\right\rangle=1=\|x\|_{M}$, so $\left\|x^{*}\right\|_{N}^{0} \geqslant 1$. Moreover, for any $y \in l_{M}$ with $\rho_{M}(y) \leqslant 1$ we have

$$
\begin{aligned}
\left\langle y, x^{*}\right\rangle & =\sum_{i=1}^{\infty} \frac{y(i) p_{i}^{-}(x(i))}{\sum_{i=1}^{\infty} x(i) p_{i}^{-}(x(i))} \leqslant \frac{\rho_{M}(y)+\rho_{N}\left(p^{-}(x)\right)}{\sum_{i=1}^{\infty} x(i) p_{i}^{-}(x(i))} \\
& \leqslant \frac{1+\rho_{N}\left(p^{-}(x)\right)}{\left.\sum_{i=1}^{\infty} x(i) p_{i}^{-} x(i)\right)}=\frac{\rho_{M}(x)+\rho_{N}\left(p^{-}(x(i))\right)}{\sum_{i=1}^{\infty} x(i) p_{i}^{-}(x(i))}=1,
\end{aligned}
$$

whence $\left\|x^{*}\right\|_{N}^{0} \leqslant 1$ and consequently $\left\|x^{*}\right\|_{N}^{0}=1$. This means that $x^{*} \in \operatorname{Grad}(x)$. Similarly $\bar{x}^{*} \in \operatorname{Grad}(x)$.

But if $x(1) \neq 0$, then $x^{*}(2) \neq \bar{x}^{*}(2)$; if $x(1)=0$, then $x^{*}(1) \neq \bar{x}^{*}(1)$. Therefore $x^{*} \neq \bar{x}^{*}$. This contradicts the assumption that $x$ is a smooth point, finishing the proof of the necessity of condition (iii-1).

If (iii-2) does not hold, we may assume that $x(1)=b_{1}, x(i)<b_{i}$ for $i \neq 1, \rho_{M}(x)=$ $1, p_{1}^{-}\left(b_{1}\right)<\infty$ and $p_{2}(x(2))>0$. Notice that

$$
\sum_{i=1}^{\infty} x(i) p_{i}^{-}(x(i))=x(1) p_{1}^{-}(x(1))+\sum_{i \neq 1} x(i) p_{i}^{-}(x(i))<\infty
$$

Let us define

$$
x^{*}(i)= \begin{cases}\frac{p_{i}^{-}(x(i))}{\sum_{i \neq 2} x(i) p_{i}^{-}(x(i))+x(2) p_{2}(x(2))} & \text { for } i \neq 2 \\ \frac{p_{2}(x(2))}{\sum_{i \neq 2} x(i) p_{i}^{-}(x(i))+x(2) p_{2}(x(2))} & \text { for } i=2 .\end{cases}
$$


Similarly as in case (iii-1) we can prove that $x^{*} \in \operatorname{Grad}(x)$. Consider also $\bar{x}^{*}$ defined by

$$
\bar{x}^{*}(i)= \begin{cases}\frac{1}{b_{1}} & \text { for } i=1 \\ 0 & \text { for } i \neq 1 .\end{cases}
$$

Then $\bar{x}^{*}(x)=1$ and for any $y \in l_{M}$ with $\rho_{M}(y) \leqslant 1$ we have $\left\langle y, \bar{x}_{1}^{*}\right\rangle=y(1) / b_{1} \leqslant b_{1} / b_{1}=$ 1. So $\left\|\bar{x}^{*}\right\|_{N}^{0}=1$, whence it follows that $\bar{x}^{*} \in \operatorname{Grad}(x)$. Note that $\bar{x}^{*} \neq x^{*}$ because $\bar{x}^{*}(2) \neq x^{*}(2)$. Therefore, $x$ cannot be smooth if (iii-2) is not satisfied.

Sufficiency. By condition (i) and Lemma 0.2, $\operatorname{Grad}(x)=R \operatorname{Grad}(x)$. Assume also that conditions (ii), (iii) are satisfied. We consider separately five cases.

I. $x(i)<b_{i}$ for all $i \in \mathbb{N}$ and $x(i)<M_{i}^{-1}(1)$ for all $i \in \mathbb{N}$.

Take an arbitrary $x^{*} \in R \operatorname{Grad}(x)$. By condition (iii-1) and Lemma 1.4, we have

$$
x^{*}(i) p_{j}^{-}(x(j))=x^{*}(i) p_{j}(x(j)) \geqslant x^{*}(j) p_{i}^{-}(x(i))=x^{*}(j) p_{i}(x(i)) \geqslant x^{*}(i) p_{j}^{-}(x(j)) .
$$

So, $x^{*}(i) p_{j}(x(j))=x^{*}(j) p_{i}(x(i))$ for every $i, j$ with $p_{i}(x(i))>0, p_{j}(x(i))>0$, whence it follows that there is $d>0$ such that

$$
\frac{x^{*}(j)}{p_{j}(x(j))}=d \quad \text { for any } j \text { with } p_{j}(x(j))>0
$$

If $x(j)<e_{j}$, we have $p_{j}(x(j))=0$ and Lemma 1.4 (i) yields $x^{*}(j)=0$. Assume that $p_{j}(x(j))=0$ and $x(j)=e_{j}$. By $\rho_{M}(x)=1$ there is $i_{0} \in \mathbb{N}$ such that $M_{i_{0}}\left(x\left(i_{0}\right)\right)>0$, so $p_{i_{0}}^{-}\left(x\left(i_{0}\right)\right)>0$. By Lemma 1.4(i),

$$
0=x^{*}\left(i_{0}\right) p_{j}(x(j)) \geqslant x^{*}(j) p_{i_{0}}^{-}\left(x\left(i_{0}\right)\right) .
$$

So, we still have $x^{*}(j)=0$. This means that $x^{*}(j)=d p_{j}(x(j))$ for all $i \in \mathbb{N}$. From $1=\left\langle x, x^{*}\right\rangle=d \sum_{i=1}^{\infty} x(i) p_{i}(x(i))$, we obtain $d=1 / \sum_{i=1}^{\infty} x(i) p_{i}(x(i))$. Hence $x^{*}(i)=$ $p_{i}(x(i)) / \sum_{i=1}^{\infty} x(i) p_{i}(x(i))$ for all $i \in \mathbb{N}$, and so $x^{*}$ is unique, that is, $x$ is smooth.

II. $x(i)<b_{i}$ for all $i \in \mathbb{N}$ and $M_{1}(x(1))=1$.

Obviously, $M_{i}(x(i))=0$ for all $i \neq 1$. So, by (iii-1) $p_{i}(x(i))=p_{i}^{-}(x(i))=0$ for all $i \neq 1$. We can prove similarly to case I that for any $x^{*} \in R \operatorname{Grad}(x)$, we have $x^{*}(i)=0$ for all $i \neq 1$. Therefore, $\operatorname{supp} x^{*}=\{1\}$, whence

$$
x^{*}=\left(\frac{1}{x(1)}, 0,0, \ldots\right)
$$

is the only element of $\operatorname{Grad}(x)$, that is, $x$ is a smooth point.

III. $x(1)=b_{1}, x(i)<b_{i}$ for $i \neq 1$ and $p_{1}^{-}\left(b_{1}\right)=\infty$.

For any $x^{*} \in R \operatorname{Grad}(x)$ and any $i \neq 1$, by Lemma 1.4 we have $x^{*}(i)=0$ if $x(i) \leqslant e_{i}$ and

$$
\infty>x^{*}(1) p_{i}^{-}(x(i)) \geqslant x^{*}(i) p_{1}^{-}(x(1)) \geqslant x^{*}(i) p_{1}^{-}\left(b_{1}\right)
$$


if $x(i) \geqslant e_{i}$. (We apply Lemma 1.4 (ii) with $j=1$ if $e_{i}=0$ and Lemma 1.4 (i) if $e_{i}>0$ ). So, we have $x^{*}(i)=0$ for $i \neq 1$. This shows that supp $x^{*}=\{1\}$, that is, $x^{*}$ is unique, namely $x^{*}=\left(b_{1}^{-1}, 0,0, \ldots\right)$.

IV. $x(1)=b_{1}, x(i)<b_{i}$ for $i \neq 1$ and $\rho_{M}(x)<1$.

For any $x^{*} \in R \operatorname{Grad}(x)$, if $x^{*}(i)>0$ for $i \neq 1$, then by $x(i)<b_{i}$, one can find $c$ with $x(i)<c<b_{i}$ satisfying $\sum_{j \neq i} M_{j}(x(j))+M_{i}(c) \leqslant 1$. Define $\bar{x}$ with $\bar{x}(i)=c$ and $\bar{x}(j)=x(j)$ for $j \neq i$. Then $\rho_{M}(\bar{x}) \leqslant 1$ and $\|\bar{x}\|_{M} \leqslant 1$. Hence

$$
1 \geqslant\left\langle\bar{x}, x^{*}\right\rangle=\left\langle x, x^{*}\right\rangle+x^{*}(i)(c-x(i))=1+x^{*}(i)(c-x(i)),
$$

which is a contradiction. So, $x^{*}(i)=0$ for $i \neq 1$, which shows that $x^{*}$ is unique (in fact $\left.x^{*}=\left(1 / b_{1}, 0,0, \ldots\right)\right)$.

V. $x(1)=b_{1}, x(i)<b_{i}$ for $i \neq 1, p_{i}(x(i))=0$ for $i \neq 1, \rho_{M}(x)=1$.

Then for any $x^{*} \in R \operatorname{Grad}(x)$, if $x^{*}(i)>0$ and $x(i) \geqslant e_{i}$ for some $i \neq 1$, by Lemma 1.4

$$
0=x^{*}(1) p_{i}(x(i)) \geqslant x^{*}(i) p_{1}\left(b_{1}\right)=x^{*}(i) p_{1}^{-}\left(M_{1}^{-1}(1)\right)>0,
$$

because $M(x(1))=1$ by $\rho_{M}(x)=1$. This is a contradiction, which shows that $x^{*}(i)=0$ for $i \neq 1$ whenever $x(i) \geqslant e_{i}$. If $0 \leqslant x(i)<e_{i}$, then $x^{*}(i)=0$ by Lemma $1.4(\mathrm{i})$. Therefore $\operatorname{supp} x^{*}=\{1\}$, whence $x^{*}$ is unique. This finishes the proof.

THEOREM 1.2. For $x \in S\left(l_{M}\right)$ the following assertions are equivalent:

(A) $x$ is a strongly smooth point,

(B) $x$ is a very smooth point,

(C) (i) $d_{M}(x)<1$,

(ii) $|x(i)|=b_{i}$ for at most one $i \in \mathbb{N}$,

(iii-1) if $|x(i)|<b_{i}$ for all $i \in \mathbb{N}$, then $N \in \delta_{2}^{0}$ and $|x(i)|<M_{i}^{-1}(1)$ implies $p_{i}^{-}(|x(i)|)=p_{i}(|x(i)|)$,

(iii-2) if $\left|x\left(i_{0}\right)\right|=b_{i_{0}}$ for some $i_{0} \in \mathbb{N}$ and $|x(i)|<b_{i}$ for $i \neq i_{o}$, then $p_{i_{0}}^{-}\left(b_{i_{0}}\right)=\infty$ or $\rho_{M}(x)<1$ or $p_{i}(|x(i)|)=0$ for $i \neq i_{0}$ and $N \in \delta_{2}^{0}$.

PRoof: We still assume without loss of generality that $x(i) \geqslant 0$ for all $i \in \mathbb{N}$. The implications $(A) \Rightarrow(B) \Rightarrow(C)(i)$,(ii) are trivial. Since very smooth points are smooth points, if (iii-1) is not true, then $N \notin \delta_{2}^{0}$ and

$$
|x(i)|<b_{i} \quad \text { for all } i \in \mathbb{N} \text {. }
$$

From Theorem 1.1, we get in this case that $\operatorname{supp} y=\operatorname{supp} x$, where $y$ determines the unique support functional of $x$. Combining this with $\rho_{M}(x)=1$, we get

$$
\sum_{i \in \text { supp } y} M_{i}\left(b_{i}\right)=\sum_{i \in \operatorname{supp} x} M_{i}\left(b_{i}\right)>\sum_{i \in \operatorname{supp} x} M_{i}(x(i))=\rho_{M}(x)=1 .
$$


By Lemma 0.5 , there is $k \geqslant 1$ such that $\|y\|_{N}^{0}=\left(1+\rho_{N}(k y)\right) / k$. Since $N \notin \delta_{2}^{0}$, there exists $z \in l_{N}^{0}$ satisfying

$$
\rho_{N}(z) \leqslant 1 \quad \text { and } \quad d_{N}\left(\frac{z}{k}-y\right) \neq 0
$$

(If $d_{N}(y)=0$ we choose $z \in l_{N}^{0}$ with $d_{N}(z)>0 ;$ if $d_{N}(y)>0$ we choose $z \in l_{N}^{0}$ with $d_{N}(z)=0$.) Let

$$
y_{n}=\left(y(1), y(2), \ldots, y(n), \frac{z(n+1)}{k}, \frac{z(n+2)}{k}, \ldots\right)
$$

Then

$$
\begin{aligned}
\left\|y_{n}\right\|_{N}^{0} & \leqslant \frac{1}{k}\left(1+\rho_{N}\left(k y_{n}\right)\right)=\frac{1}{k}\left(1+\sum_{i \leqslant n} N_{i}(k y(i))+\sum_{i>n} N_{i}(z(i))\right) \\
& \leqslant \frac{1}{k}\left(1+\rho_{N}(k y)\right)+\sum_{i>n} N_{i}(z(i)) \rightarrow\|y\|_{N}^{0}=1 .
\end{aligned}
$$

So $\limsup _{n \rightarrow \infty}\left\|y_{n}\right\|_{N}^{0} \leqslant 1$. On the other hand

$$
\left\langle x, y_{n}\right\rangle=\sum_{i=1}^{n} x(i) y_{n}(i)+\frac{1}{k} \sum_{i=n+1}^{\infty} x(i) z(i)
$$

and by

$$
\frac{1}{k} \sum_{i>n} x(i) z(i) \leqslant \frac{1}{k} \sum_{i=n+1}^{\infty}\left(M_{i}(x(i))+N_{i}(z(i))\right) \rightarrow 0,
$$

we have $\left\langle x, y_{n}\right\rangle \rightarrow\langle x, y\rangle=1$. So, $\liminf _{n \rightarrow \infty}\left\|y_{n}\right\|_{N}^{0} \geqslant 1$. Hence $\lim _{n \rightarrow \infty}\left\|y_{n}\right\|_{N}^{0}=1$. But since $d_{N}((z / k)-y) \neq 0$, there is a singular functional $\varphi$ such that $\varphi((z / k)-z) \neq 0$. Thus

$$
\varphi\left(y_{n}-y\right)=\varphi\left(\frac{z}{k}-y\right) \neq 0, \text { that is, } y_{n} \nrightarrow^{\mathrm{w}} y \text { as } n \rightarrow \infty
$$

This contradicts the fact that $x$ is a very smooth point.

If (iii-2) does not hold, then we may assume that $x(1)=b_{1}, x(i)<b_{i}$ for all $i \neq 1, \rho_{M}(x)=1, p_{1}^{-}\left(b_{1}\right)<\infty$ and $N \notin \delta_{2}^{0}$. Since $x$ is a smooth point, by condition (iii-2) from Theorem 1.1, we have $p_{i}(x(i))=0$ for $i \neq 1$. So, $M_{i}(x(i))=0$ for $i \neq 1$ and $M_{1}\left(x(1)=\rho_{M}(x)=1\right.$. In this case, $y=\left(b_{1}^{-}, 0,0, \ldots\right)$ is the only support functional at $x$. Take $k>0$ satisfying $k / b_{1}=p_{1}^{-}\left(b_{1}\right)$. Then

$$
\frac{1}{k}\left(1+\rho_{N}(k y)\right)=\frac{1}{k}\left(M_{1}\left(b_{1}\right)+N_{1}\left(\frac{k}{b_{1}}\right)\right)=\frac{1}{k}\left(b_{1} \cdot \frac{k}{b_{1}}\right)=1=\|y\|_{N}^{0} .
$$

Now, we can deduce a contradiction in the same way as above, proving the necessity of condition (iii-2). 
(C) $\Rightarrow(A)$. It follows from Theorem 1.1 that $x$ is a smooth point, so $x$ has a unique support functional $y$. Suppose that $f_{n} \in S\left(l_{M}^{*}\right), f_{n}(x) \rightarrow 1$ and $f_{n}=y_{n}+\varphi_{n}$, where $y_{n} \in l_{N}^{0}$ and $\varphi_{n}$ are singular functionals for all $n \in \mathbb{N}$. We need to prove that $\left\|f_{n}-y\right\| \rightarrow 0$ as $n \rightarrow \infty$. By $d_{M}(x)<1$ and Lemma 0.1, there is $z \in h_{M}$ such that $\|x-z\|<1-\theta<1$. Thus

$$
\begin{aligned}
1 \leftarrow f_{n}(x) & =\left\langle x, y_{n}\right\rangle+\varphi_{n}(x) \leqslant\|x\|_{M}\left\|y_{n}\right\|_{N}^{0}+\varphi_{n}(x-z) \\
& \leqslant\left\|y_{n}\right\|_{N}^{0}+\left\|\varphi_{n}\right\|\|x-z\|_{M}<\left\|y_{n}\right\|_{N}^{0}+\left\|\varphi_{n}\right\|(1-\theta) \\
& =\left\|f_{n}\right\|-\theta\left\|\varphi_{n}\right\|=1-\theta\left\|\varphi_{n}\right\|,
\end{aligned}
$$

whence $\left\|\varphi_{n}\right\| \rightarrow 0$ as $n \rightarrow \infty$. Therefore, we can assume in the rest of the proof of this implication that $\left\|y_{n}\right\|_{N}^{0}=1$ and $\left\langle x, y_{n}\right\rangle \rightarrow 1$ as $n \rightarrow \infty$. By $d_{M}(x)<1$ and Lemma 1.2, we have

$$
\lim _{i_{0} \rightarrow \infty} \sup _{n} \sum_{i>i_{0}} N_{i}\left(y_{n}(i)\right)=0 .
$$

By (6) and Young's equality, we get

$$
\lim _{i_{0} \rightarrow \infty} \sup _{n} \sum_{i>i_{0}} x(i) y_{n}(i)=0 .
$$

To show that $\left\|y_{n}-y\right\| \rightarrow 0$ as $n \rightarrow \infty$, we consider the following five cases.

I. $x(i)<b_{i}$ and $x(i)<M_{i}^{-1}(1)$ for all $i \in \mathbb{N}$.

From (C) (iii-1), we get $p_{i}^{-}(x(i))=p_{i}(x(i))$ for all $i \in \mathbb{N}$ and $N \in \delta_{2}^{0}$. By Theorem 1.1 , the unique support functional $y$ at $x$ is given by

$$
y=\frac{1}{\sum_{i=1}^{\infty} x(i) p_{i}(x(i))}\left(p_{1}\left(x_{1}\right), p_{2}\left(x_{2}\right), \ldots\right) .
$$

From Lemma 1.3(ii), in the case when $p_{i}(x(i))>0$ and $p_{j}(x(j))>0$, we have

$$
\lim _{n \rightarrow \infty} p_{i}(x(i)) y_{n}(j)=\lim _{n \rightarrow \infty} p_{j}(x(j)) y_{n}(i) .
$$

So, there exists $d>0$ such that

$$
\lim _{n \rightarrow \infty} y_{n}(j)=d p_{j}(x(j))
$$

for every $j \in \mathbb{N}$ with $p_{j}(x(j))>0$ (namely $d$ is the common value of $\lim _{n \rightarrow \infty}\left(y_{n}(i) / p(x(i))\right.$ ) which does not depend on $i$ ).

Assume now that $p_{j}(x(j))=0$. If $x(j)<e_{j}$, then $y_{n}(j) \rightarrow 0$ by Lemma 1.3(i). If $x(j)=e_{j}$ and $y_{n}(j) \nrightarrow 0$ as $n \rightarrow \infty$, we can assume that $y_{n}(j) \geqslant c>0$ for all $n \in \mathbb{N}$. There exists $i_{0} \in \mathbb{N}$ such that $p_{i_{0}}^{-}\left(x\left(i_{0}\right)\right)>0$ (otherwise $p_{i}^{-}(x(i))=0$ and $M_{i}(x(i))=0$ 
for all $i \in \mathbb{N}$, whence $\rho_{M}(x)=0 \neq 1$, a contradiction to (4)). Therefore $p_{i_{0}}^{-}\left(x\left(i_{0}\right)\right)>0$ for some $i_{0} \in \mathbb{N}$ and consequently

$$
\lim _{n \rightarrow \infty}\left(y_{n}\left(i_{0}\right) p_{j}(x(j))-y_{n}(j) p_{i_{0}}^{-}\left(x\left(i_{0}\right)\right)\right)<0,
$$

which contradicts Lemma $1.4(\mathrm{i})$. So, $y_{n}(j) \rightarrow 0$ as $n \rightarrow \infty$. This means that

$$
y_{n}(j) \rightarrow d p_{j}(x(j))
$$

for any $j \in \mathbb{N}$. Combining this with (7), we get

$$
1 \leftarrow\left\langle x, y_{n}\right\rangle=\sum_{i=1}^{\infty} x(i) y_{n}(i) \rightarrow d \sum_{i=1}^{\infty} x(i) p_{i}(x(i))
$$

whence

$$
d=1 / \sum_{i=1}^{\infty} x(i) p_{i}(x(i))
$$

This implies that $y_{n}(j)-y(j) \rightarrow 0$ as $n \rightarrow \infty$ for all $j \in \mathbb{N}$. Combining this with (6), we get

$$
\lim _{i_{0} \rightarrow \infty} \sup _{n} \sum_{i>i_{0}} N_{i}\left(\frac{y_{n}(i)-y(i)}{2}\right)=0 .
$$

By Lemma 1.1, $\left\|\left(y_{n}-y\right) / 2\right\|_{N}^{0} \rightarrow 0$, that is, $\left\|y_{n}-y\right\|_{N}^{0} \rightarrow 0$ as $n \rightarrow \infty$.

II. $x(i)<b_{i}$ for all $i \in \mathbb{N}$ and $x(1)=M_{1}^{-1}(1)$.

In this case it follows from condition (C) (iii-1) that $N \in \delta_{2}^{0}$ and $p_{i}^{-}(x(i))=$ $p_{i}(x(i))=0$ for $i \neq 1$ (since $M_{i}(x(i))=0$ and $p_{i}$ is continuous at $x(i)$ for $i \neq 1$ ). It follows from the proof of Theorem 1.1 that the unique support functional at $x$ is represented by the sequence

$$
y=\left(\frac{1}{x(1)}, 0,0, \ldots\right)
$$

In a similar way to case I, we can prove that $y_{n}(i) \rightarrow 0$ for $i \neq 1$. By (7), we get

$$
1=\lim _{n \rightarrow \infty} \sum_{i=1}^{\infty} x(i) y_{n}(i)=\lim _{n \rightarrow \infty} x(1) y_{n}(1)
$$

Therefore, $y_{n}(1) \rightarrow 1 / x(1)=y(1)$. From (6) and Lemma 1.1, we get $\left\|y_{n}-y\right\|_{N}^{0} \rightarrow 0$ as $n \rightarrow \infty$.

III. $x(1)=b_{1}, x(i)<b_{i}$ for $i \neq 1, p_{1}^{-}\left(b_{1}\right)<\infty$ and $\rho_{M}(x)=1$.

By (C)(iii-2), we get $p_{i}(x(i))=0$ for $i \neq 1$ and $N \in \delta_{2}^{0}$. From the proof of Theorem 1.1 it follows that the support functional at $x$ is given by $y=\left(\left(1 / b_{1}\right), 0,0, \ldots\right)$. Similarly to case I we can prove that $y_{n}(i) \rightarrow 0$ as $n \rightarrow \infty$ for all $i \neq 1$. By (7), we get $y_{n}(1) \rightarrow 1 / b_{1}=y(1)$. From (6) and Lemma 1.1 it follows that $\left\|y_{n}-y\right\|_{N}^{0} \rightarrow 0$ as $n \rightarrow \infty$.

IV. $x(1)=b_{1}, x(i)<b_{i}$ for $i \neq 1$ and $p_{1}^{-}\left(b_{1}\right)=\infty$. 
In this case, $N \in \delta_{2}^{0}$ is not necessary. We get from the proof of Theorem 1.1 that the unique support functional at $x$ is given by $y=\left(1 / b_{1}, 0,0, \ldots\right)$.

If $x(i)<e_{i}$, by Lemma $1.3(\mathrm{i})$, we get $y_{n}(i) \rightarrow 0$ as $n \rightarrow \infty$. If $x(i) \geqslant e_{i}$, by Lemma $1.3(\mathrm{ii})$,

$$
\lim _{n \rightarrow \infty}\left(y_{n}(1)\right) p_{i}(x(i))-y_{n}(i) p_{1}^{-}(x(1)) \geqslant 0 .
$$

But $p_{i}(x(i))<\infty, y_{n}(1) \leqslant N_{1}^{-1}(1), y_{n}(1) p_{i}(x(i))<\infty$. So, $y_{n}(i) \rightarrow 0$ as $n \rightarrow \infty$ for $i \neq 1$. By $(7)$,

$$
y_{n}(1) \rightarrow \frac{1}{b_{1}}=y(1), \quad \text { that is, } \quad y_{n}(i) \rightarrow y(i) \quad(i=1,2, \ldots) .
$$

Now, we shail consider two subcases to finish the proof in this case. By (7),

IV.1. For an infinite number of $n$ we have $1=\|y\|_{N}^{0}=\sum_{i=1}^{\infty} y_{n}(i) b_{i}$.

$$
1=\lim _{n \rightarrow \infty} \sum_{i=1}^{\infty} x(i) y_{n}(i)=\lim _{n \rightarrow \infty} x(1) y_{n}(1)=x(1) / b_{1}
$$

Hence $\lim _{n \rightarrow \infty} \sum_{i=2}^{\infty} y_{n}(i) b_{i}=0$. Consequently

$$
\lim _{n \rightarrow \infty}\left\|y_{n}-y\right\|_{N}^{0}=\lim _{n \rightarrow \infty}\left\|\left(0, y_{n}(2), y_{n}(3), \ldots\right)\right\|_{N}^{0} \leqslant \lim _{n \rightarrow \infty} \sum_{i=2}^{\infty} y_{n}(i) b_{i}=0 .
$$

IV.2. There is an infinite number of $n$ for which

$$
1=\left\|y_{n}\right\|_{N}^{0}=\frac{1}{k_{n}}\left(1+\rho_{N}\left(k_{n} y_{n}\right)\right)
$$

First, we shall prove that $k_{n} \rightarrow \infty$. Otherwise, we can assume (passing to a subsequence if necessary) that $k_{n} \rightarrow k_{0}<\infty$. Since $k_{0} / b_{1}<\infty=p_{1}^{-}\left(b_{1}\right)$, there exists $c>0$ such that $M_{1}\left(b_{1}\right)+N_{1}\left(k_{0} / b_{1}\right) \geqslant b_{1}\left(k_{0} / b_{1}\right)+c=k_{0}+c$. Hence

$$
\begin{aligned}
1 & =\lim _{n \rightarrow \infty} \frac{1}{k_{n}}\left(1+\rho_{N}\left(k_{n} y_{n}\right)\right) \geqslant \lim _{n \rightarrow \infty} \frac{1}{k_{n}}\left(1+N_{1}\left(k_{n} y_{n}(1)\right)\right) \\
& =\frac{1}{k_{0}}\left(1+N_{1}\left(k_{0} / b_{1}\right)\right) \geqslant \frac{1}{k_{0}}\left(M_{1}\left(b_{1}\right)+N_{1}\left(k_{0} / b_{1}\right)\right) \geqslant \frac{k_{0}+c}{k_{0}}=1+\frac{c}{k_{0}} .
\end{aligned}
$$

This is a contradiction. Notice that

$$
\lim _{n \rightarrow \infty} \frac{N_{1}\left(k_{n} y_{n}(1)\right)}{k_{n}} \geqslant \lim _{n \rightarrow \infty} \frac{N_{1}\left(k_{n} / b_{1}\right)}{k_{n}} \geqslant \lim _{n \rightarrow \infty} \frac{\left(k_{n} / b_{1}\right) \cdot b_{1}-M_{1}\left(b_{1}\right)}{k_{n}}=1,
$$

whence

$$
1=\lim _{n \rightarrow \infty} \frac{1}{k_{n}}\left(1+\rho_{N}\left(k_{n} y_{n}\right)\right) \geqslant 1+\lim _{n \rightarrow \infty} \frac{1}{k_{n}} \sum_{i=2}^{\infty} N_{i}\left(k_{n} y_{n}(i)\right)
$$


So, $\lim _{n \rightarrow \infty}\left(1 / k_{n}\right) \sum_{i=2}^{\infty} N_{i}\left(k_{n} y_{n}(i)\right)=0$. Consequently

$$
\begin{aligned}
\lim _{n \rightarrow \infty}\left\|y_{n}-y\right\|_{N}^{0} & =\lim _{n \rightarrow \infty}\left\|\left(0, y_{n}(2), y_{n}(3), \ldots\right)\right\|_{N}^{0} \\
& \leqslant \lim _{n \rightarrow \infty} \frac{1}{k_{n}}\left(1+\sum_{i=2}^{\infty} N_{i}\left(k_{n} y_{n}(i)\right)\right)=0 .
\end{aligned}
$$

V. $x(1)=b_{1}, x(i)<b_{i}$ for $i \neq 1$ and $\rho_{M}(x)<1$.

By the proof of Theorem 1.1, the unique support functional at $x$ is given by $y=$ $\left(1 / b_{1}, 0,0, \ldots\right)$. Assume to the contrary that $\left(y_{n}\right)$ does not converge coordinatewise for $i \neq 1$. Then we can assume that there is $i \neq 1$ such that $y_{n}(i) \geqslant c>0$ for all $n \in \mathbb{N}$. Since $x(i)<b_{i}$, one can find $u>0$ with $x(i)<u<b_{i}$ satisfying $\sum_{j \neq i} M_{j}(x(j))+M_{i}(u) \leqslant 1$. Define $\bar{x}$ with $\bar{x}(j)=x(j)$ for $j \neq i$ and $\bar{x}(i)=u$. Then $\rho_{M}(\bar{x}) \leqslant 1$. But

$$
\begin{aligned}
\left\langle\bar{x}, y_{n}\right\rangle & =\left\langle x, y_{n}\right\rangle+y_{n}(i)(u-x(i)) \geqslant\left\langle x, y_{n}\right\rangle+c(u-x(i)) \\
& \rightarrow 1+c(u-x(i)) .
\end{aligned}
$$

This contradicts the inequality $\left\langle\bar{x}, y_{n}\right\rangle \leqslant\|\bar{x}\|_{M}\left\|y_{n}\right\|_{N}^{0}=1$. So, $y_{n}(i) \rightarrow 0$ as $n \rightarrow \infty$ for $i \neq 1$. Using (7), we get $y_{n}(1) \rightarrow y(1)=1 / b_{1}$. We divide the remaining part of the proof into two subcasses.

$\mathrm{V}-1 .\left\|y_{n}\right\|_{N}^{0}=\sum_{i=1}^{\infty} b_{i} y_{n}(i)$ for an infinite number of $n$.

Similarly to case IV-1 we can prove that $\left\|y_{n}-y\right\|_{N}^{0} \rightarrow 0$ as $n \rightarrow \infty$.

V-2. $\left\|y_{n}\right\|_{N}^{0}=\left(1 / k_{n}\right)\left(1+\rho_{N}\left(k_{n} y_{n}\right)\right)$ for an infinite number of $n$.

We shall show that in this case $\lim \sup k_{n}=\infty$. Otherwise, we can assume that $k_{n} \rightarrow$ $k_{0}<\infty$, whence

$$
\begin{aligned}
1 & =\lim _{n \rightarrow \infty} \frac{1}{k_{n}}\left(1+\rho_{N}\left(k_{n} y_{n}\right)\right) \geqslant \lim _{n \rightarrow \infty} \frac{1}{k_{n}}\left(1+N_{1}\left(k_{n} y_{n}(1)\right)\right) \\
& =\frac{1}{k_{0}}\left(1+N_{1}\left(\frac{k_{0}}{b_{1}}\right)\right)=\frac{1}{k_{0}}\left(M_{1}\left(b_{1}\right)+N_{1}\left(\frac{k_{0}}{b_{1}}\right)+1-M_{1}\left(b_{1}\right)\right) \\
& \geqslant \frac{1}{k_{0}}\left(b_{1} \cdot \frac{k_{0}}{b_{1}}+1-\rho_{M}(x)\right)=1+\frac{1-\rho_{M}(x)}{k_{0}},
\end{aligned}
$$

a contradiction, which shows that $\lim \sup k_{n}=\infty$. Now, we can prove that $\left\|y_{n}-y\right\|_{N}^{0} \rightarrow 0$ similarly to case IV-2.

As an immediate consequence of Theorem 1.2 we get the following:

COROLLARY 1.1. The following assertions are equivalent:

(i) $l_{M}$ is strongly smooth,

(ii) $l_{M}$ is very smooth,

(iii) $l_{M}$ is smooth and $N \in \delta_{2}^{0}$. 


\section{REFERENCES}

[1] S. Chen, Geometry of Orlicz spaces, Dissertationes Math. 356, (1996).

[2] S. Chen, 'Smoothness of Orlicz spaces', Comment. Math. Prace Mat. 27 (1987), 49-58.

[3] S. Chen, H. Hudzik and A. Kamińska, 'Support functionals and smooth points in Orlicz function spaces equipped with the Orlicz norm', Math. Japon. 39 (1992), 271-279.

[4] S. Chen, H. Hudzik and M. Wista, 'Smooth points in Orlicz sequence spaces and geometry of the dual and bidual of Orlicz spaces', Math. Japon. 44 (1996), 367-375.

[5] J. Diestel, Geometry of Banach spaces - selected topics, Lecture Notes in Math. 485 (Springer-Verlag, Berlin, Heidelberg, New York, 1975).

[6] R. Grząślewicz and H. Hudzik, 'Smooth points of Orlicz spaces equipped with Luxemburg norm', Math. Nachr. 155 (1992), 31-45.

[7] H. Hudzik and Y. Ye, 'Support functionals and smoothness in Musielak-Orlicz sequence space equipped with Luxemburg norm', Comment. Math. Univ. Carolin. 31 (1990), 661-684.

[8] H. Hudzik and Z. Zba̧szyniak, 'Smooth points of Musielak-Orlicz sequence spaces equipped with the Luxemburg norm', Collog. Math. 65 (1993), 157-164.

[9] H. Hudzik and Z. Zbagszyniak, 'Smoothness in Musielak-Orlicz spaces', Collect. Math. 48 (1997), 543-561.

[10] M. A. Krasnoselskii and Ya. B. Rutickii, Convex functions and Orlicz spaces, (translation) (Nordhoff, Groningen, 1961).

[11] J. Lindenstrauss and L. Tzafriri, Classical Banach spaces I, Lecture Notes in Math. (Springer-verlag, Berlin, Heidelberg, New York, 1977).

[12] W.A.J. Luxemburg, Banach function spaces, Thesis (Delft, 1955).

[13] L. Maligranda, Orlicz spaces and interpolation, Seminars in Math. 5 (Campinas, 1989).

[14] J. Musielak, Orlicz spaces and modular spaces, Lecture Notes in Math. 1034 (Springer-Verlag, Berlin, Heidelberg, New York, Tokyo, 1983).

[15] M.M. Rao and Z.D. Ren, Theory of Orlicz spaces, Monographs and Textbooks in Pure and Applied Mathematics 146 (Marcel Dekker, New York, 1991).

[16] C. Wu and H. Sun, 'The norm calculation and convexities in Musielak-Orlicz spaces', Chinese Ann. Math. 12 (1991), 98-102.

[17] Z. Zbasszyniak, 'Smoothness of Musielak-Orlicz sequence spaces equipped with the Orlicz norm', in Proceed. of the Fifth Intern. Conf. on Function Spaces (Marcel Dekker) (to appear).

Department of Mathematics

Harbin Normal University

Harbin

People's Republic of China

Department of Mathematics

Harbin University of Science and Technology

PO Box 123

Harbin 150080

People's Republic of China
Faculty of Mathematics and Computer Science Adam Mickiewicz University

Poznań

Poland

and

Institute of Mathematics

Poznań University of Technology

Poznań

Poland 